\title{
Mobile Technology-Based (mLearning) Intervention to Enhance Breast Cancer Clinicians' Communication About Sexual Health: A Pilot Trial
}

\author{
Jennifer Barsky Reese, $\mathrm{PhD}^{1}$; Lauren A. Zimmaro, $\mathrm{PhD}^{1}$; Sharon L. Bober, $\mathrm{PhD}^{2,3}$; Kristen Sorice, $\mathrm{BA}^{1}$; \\ Elizabeth Handorf, $\mathrm{PhD}^{1,4}$; Elaine Wittenberg, $\mathrm{PhD}^{5}$; Areej El-Jawahri, $\mathrm{MD}^{6}$; Mary Catherine Beach, MD, MPH ; \\ Antonio C. Wolff, MD ${ }^{8}$; Mary B. Daly, MD, $\mathrm{PhD}^{9}$; Brynna Izquierdo, $\mathrm{MPH}^{10}$; and Stephen J. Lepore, $\mathrm{PhD}^{1,11}$
}

\begin{abstract}
Background: Most breast cancer clinicians lack training to counsel patients about sexual concerns. The purpose of this study was to assess the feasibility, acceptability, and preliminary effects of a mobile learning (mLearning) intervention (improving Sexual Health and Augmenting Relationships through Education [iSHARE]) aimed at enhancing breast cancer clinicians' knowledge of, beliefs about, and comfort with discussing patients' sexual health concerns. Methods: Clinicians listened to a 2-part educational podcast series offering information on breast cancer-related sexual health concerns and effective communication on the topic, which consisted of interviews with expert guests. Intervention feasibility was assessed through rates of enrollment, retention, and intervention completion, with benchmarks of $40 \%$, $70 \%$, and $60 \%$, respectively. Acceptability was assessed through program evaluations, with $75 \%$ of clinicians rating the intervention favorably (eg, relevance, satisfaction) signifying acceptability. Clinicians self-reported their knowledge about breast cancer-related sexual health concerns, beliefs (ie, self-efficacy for discussing sexual health concerns), and comfort with discussing sexual concerns measured at preintervention and postintervention. Qualitative analysis examined clinicians' perceptions of lessons learned from the intervention. Results: A total of 32 breast cancer clinicians enrolled (46\% of those invited; $97 \%$ of those who responded and screened eligible), 30 (94\%) completed both the intervention and study surveys, and $80 \%$ rated the intervention favorably, demonstrating feasibility and acceptability. Results showed positive trends for improvement in clinician knowledge, beliefs, and comfort with discussing sexual health concerns. Clinicians reported key lessons learned, including taking a proactive approach to discussing sexual health concerns, normalizing the topic, addressing vaginal health, sending the message that help is available, and assessing sexual health concerns with patients from different backgrounds. Conclusions: Breast cancer clinicians were amenable to participating in the iSHARE intervention and found it useful. iSHARE showed promise for improving clinician's knowledge and comfort discussing patients' sexual health concerns. A larger trial is required to demonstrate efficacy. Future studies should also examine whether iSHARE can improve patient-clinician communication and address patients' sexual concerns.
\end{abstract}

J Natl Compr Canc Netw 2021;19(10):1133-1140 doi: 10.6004/jnccn.2021.7032

\section{Background}

Breast cancer negatively affects patients' sexuality and intimacy, leading to physical (eg, vaginal dryness) and emotional problems (eg, body image distress). ${ }^{1-6}$ These sexual problems tend to persist beyond initial treatment even as other quality of life (QoL) concerns improve. ${ }^{1,7,8}$ Guidelines set forth by NCCN and ASCO urge breast cancer clinicians to discuss treatment-related sexual adverse effects with their patients in their routine cancer care, ${ }^{9-11}$ and most women diagnosed with breast cancer want clinicians to discuss these issues as part of their care. ${ }^{12,13}$ However, only $30 \%$ to $40 \%$ of women report that their clinicians discussed sexual concerns with them. ${ }^{13,14}$ A key factor underlying this lack of communication is a lack of training in discussing sexual health and related concerns. ${ }^{15-17}$

Previously, we tested a brief intervention aimed at enhancing breast cancer clinicians' communication regarding their patients' sexual health concerns, called improving Sexual Health and Augmenting Relationships through Education (iSHARE). ${ }^{18}$ The intervention consisted of education and skills training using a self-study workbook followed by a 1-hour in-person small group workshop. In a pilot study involving 7 clinicians and 134 patients with breast cancer, positive effects were seen in clinicians' communication about sexual health concerns

${ }^{1}$ Cancer Prevention and Control Program, Fox Chase Cancer Center, Philadelphia, Pennsylvania; ${ }^{2}$ Department of Psychosocial Oncology and Palliative Care, Dana-Farber Cancer Institute, Boston, Massachusetts; ${ }^{3}$ Division of Palliative Medicine, Department of Medicine, Brigham and Women's Hospital, Boston, Massachusetts; ${ }^{4}$ Biostatistics and Bioinformatics Facility, Fox Chase Cancer Center, Philadelphia, Pennsylvania; ${ }^{5}$ Department of

Communication Studies, California State University, Los Angeles, Los Angeles, California; ${ }^{6}$ Department of Hematology/Oncology, Massachusetts General Hospital, Boston, Massachusetts; ${ }^{7}$ Department of Medicine, Johns Hopkins School of Medicine, Baltimore, Maryland; ${ }^{8}$ Women's Malignancies Program, Johns Hopkins Sidney Kimmel Comprehensive Cancer Center, Baltimore, Maryland; ${ }^{9}$ Department of Clinical Genetics, Fox Chase Cancer Center, Philadelphia, Pennsylvania; and ${ }^{10}$ Department of Epidemiology and Biostatistics, and ${ }^{11}$ Department of Social and Behavioral Sciences, College of Public Health, Temple University, Philadelphia, Pennsylvania. 
measured during routine clinic encounters, as well as in their confidence, or self-efficacy, with engaging in such communication. However, although the content was well received, the in-person group format presented challenges for recruitment and implementation and would require significant resources to be disseminated.

To address these limitations while leveraging the potential of iSHARE, we aimed to adapt this intervention to a mobile-accessible (mLearning) format and pilot test it. The present study thus investigated the feasibility, acceptability, and preliminary effects of the new iSHARE mLearning intervention, consisting of an educational 2-part podcast series, on breast cancer clinicians' knowledge of, beliefs about, and comfort with discussing patients' sexual health concerns in a pilot study. We also explored clinicians' perspectives on the key lessons they gained from the intervention (ie, lessons learned) through a qualitative analysis.

\section{Methods}

\section{Research Design and Setting}

A single-arm repeated measures design with baseline and postintervention measures was used to meet study objectives. This study was approved by the Institutional Review Board at Fox Chase Cancer Center (\#18-1068) and was entered prospectively on ClinicalTrials.gov (identifier: NCT04262219).

\section{Participants}

Medical oncologists or oncology advanced practice clinicians treating patients with breast cancer were eligible to participate. Clinicians who had participated in the trial of the iSHARE intervention in its initial format were excluded. ${ }^{18}$

\section{Recruitment and Procedures}

Clinicians were recruited from Fox Chase Cancer Center, community hospitals, and other institutions either during staff meetings or through direct contact with the principal investigator or colleagues. Introductory emails were sent to potentially eligible clinicians and included links to a REDCap $^{19,20}$ form asking clinicians to provide contact information and complete an eligibility screening questionnaire. Automated links for eligible participants were then provided for completing online consent and, after consent, the baseline survey. Participants were mailed $\$ 50$ gift cards for completing baseline and postintervention surveys ( $\$ 100$ total).

\section{iSHARE Intervention}

The iSHARE intervention content was informed by formative qualitative research, ${ }^{21}$ models of clinical communication skills development, ${ }^{22-24}$ and social cognitive theory, which emphasizes individuals' beliefs (ie, self-efficacy and outcome expectancies) as critical processes underlying successful behavioral interventions..$^{22,25-28}$ We adapted the intervention from the previous iSHARE format (written materials and in-person small group workshop) ${ }^{18}$ to the new mLearning format using expert review and structured cognitive interviews with medical oncology fellows that provided feedback on scripts guiding interviews featured in the podcast series. ${ }^{29}$ The resulting intervention consisted of a 2-part podcast series featuring 2 episodes with 30-minute one-on-one interviews with expert guests led by the principal investigator (J.B. Reese) in a semistructured question-and-answer format (see Table 1 for content description). Episodes were distributed to clinicians through the REDCap platform; clinicians could listen on any device by clicking on a link emailed to them (smartphone, computer, tablet).

\section{Data Collection}

Data were collected from participants after consent (baseline) and after intervention completion (postintervention); acceptability items and knowledge items were asked after each episode rather than at postintervention in order to assess clinicians' perspectives of the episode-specific content and accurately gauge intervention effects on knowledge.

\section{Measures}

\section{Feasibility}

Feasibility was measured through rates of enrollment (ie, percent of eligible participants approached who enrolled), retention to study completion (ie, percent of consented participants who completed all study surveys), and intervention completion (ie, percent of participants who indicated that they listened to both podcast episodes). Benchmarks for enrollment ( $\geq 40 \%$ of eligible participants), retention ( $\geq 70 \%$ ), and intervention completion $(>60 \%)$ were based on our previous iSHARE study ${ }^{18}$ but were lowered slightly because we were recruiting mostly clinicians external to the study site.

\section{Acceptability}

Acceptability was measured through standard self-report items assessing clinicians' perspectives of the intervention content and format (eg, satisfaction, relevance, ease of listening, likelihood of recommending to colleagues, likelihood of impacting practice). Responses were on a 5-point scale ranging from "very" to "not at all." The benchmark for acceptability was met if $75 \%$ of participants in the sample endorsed at least $75 \%(9 / 12)$ of the acceptability items favorably ("quite a bit" or "very"). In addition, a single open-ended question asked clinicians for the "one takeaway message" they received from each of the 2 


\section{Table 1. Content of iSHARE Intervention}

Overarching learning objectives

- Gain knowledge of sexual problems for women with breast cancer to provide a foundation for effective communication about sexual issues

- Enhance beliefs (eg, confidence) and comfort for clinical communication about sexual issues for women with breast cancer with a focus on raising the topic with patients and engaging in inquiry

\section{Major topics covered}

- Breast cancer-related sexual problems (eg, rates, types, causes)

- Women's sexual response (eg, desire, arousal, pain/discomfort)

- Treatments/solutions for addressing sexual problems, with specific information on vaginal health strategies (eg, lubricants, moisturizers)

- Role and expectations of clinician in discussing sexual health concerns with patients

- Considerations and strategies for initiating the clinical conversation (eg, when to raise topic, suggested phrasings)

- Considerations regarding referring patients for further assessment/treatment

- Case studies of common scenarios for discussing sexual health concerns for women after breast cancer (ie, discussing potential sexual adverse effects for a patient beginning chemotherapy; discussing sexual problems for women on aromatase inhibitors)

Abbreviation: iSHARE, improving Sexual Health and Augmenting Relationships through Education.

podcast episodes (ie, lessons learned); these data were analyzed qualitatively.

\section{Knowledge}

Knowledge about sexual response/breast cancer-related sexual adverse effects, common approaches and resources, and necessary communication skills for addressing sexual issues were assessed using 10 multiple-choice items adapted from those used in prior studies ${ }^{30-32}$ through expert review; an equal number of items assessed knowledge relevant to each of the 2 episodes. The sum of correct responses was used.

\section{Beliefs}

Items developed for the initial iSHARE study based on social cognitive theory ${ }^{25,33}$ assessed self-efficacy for communicating with patients with breast cancer about sexual health concerns (3 items), and outcome expectancies for communicating with patients with breast cancer about sexual health concerns (7 items), which are rated on an 11-point scale $(0=$ not at all confident/not at all; $10=$ extremely confident/very much). Mean scores across all items on these scales were used, and the internal reliability was excellent (Cronbach $\alpha \geq 0.90$ ).

\section{Comfort}

Comfort with discussing sexual health concerns was assessed using 7 items (eg, asking the patient about any sexual concerns, discussing the patient's loss of sexual interest or desire) adapted from items used in other communication studies with cancer clinicians ${ }^{34}$ using expert input and formative research on this topic. ${ }^{21}$ Responses were rated on an 11-point scale $(0=$ not at all comfortable; $10=$ extremely comfortable). Mean scores across the 7 items were used in analyses and the items had excellent reliability (Cronbach $\alpha=0.95$ ).

\section{Analytic Plan}

Data analysis was conducted using SPSS Statistics, version 24 (IBM Corp), using descriptive analyses to summarize the feasibility and acceptability of the intervention. Open-ended responses were analyzed using thematic analysis, with responses grouped with others based on thematic similarity into key lessons learned categories, and those categories subsumed under more comprehensive themes. A primary coder conducted the first round of coding (J.B. Reese) and a second coder (S.L. Bober) reviewed these codes, with consensus achieved through discussion. As per recent recommendations, ${ }^{35}$ preliminary outcome data were analyzed descriptively, with $95 \%$ confidence intervals calculated to summarize the data at the 2 assessments. A sample size of 30 clinicians completing the study was determined a priori to be adequate to achieve study objectives; this sample size was chosen to maximize the chance of correctly determining feasibility and acceptability (ie, maximize the chance of true-positive results and minimize the chance of false-positive results).

\section{Results}

\section{Participants}

Characteristics are presented for the 30 clinicians who completed the study. Of these clinicians, 20 (67\%) were medical oncologists, 7 (23\%) were nurse practitioners, and $3(10 \%)$ were physician assistants. The mean [SD] age of participants was 42.5 [10.0] years (range, 24-61 years). Most clinicians $(\mathrm{n}=22 ; 73 \%)$ identified as White, 6 (20\%) identified as Asian/Southeast Asian, 1 (3\%) identified as Black, and 1 (3\%) identified as other; 3 clinicians (10\%) identified as Hispanic/Latino. A total of 22 clinicians (73\%) identified as female. Regarding years in practice, 9 (30\%) had $\leq 5$ years, 7 (23\%) had 6 to 10 years, $6(20 \%)$ had between 11 and 15 years, and 8 (27\%) had $>15$ years.

\section{Feasibility}

Enrollment took place between February and October 2020. Of the 70 clinicians approached to participate, 33 completed the eligibility screen, whereas the remainder did not respond to the invitation (Figure 1). All 33 who completed the eligibility screen were eligible to participate in the study, and 32 consented. Of these, 30 listened to both episodes and completed the postintervention study survey. Rates of enrollment ( $46 \%$ of those invited; $97 \%$ of those screened eligible), intervention completion (94\%), and 


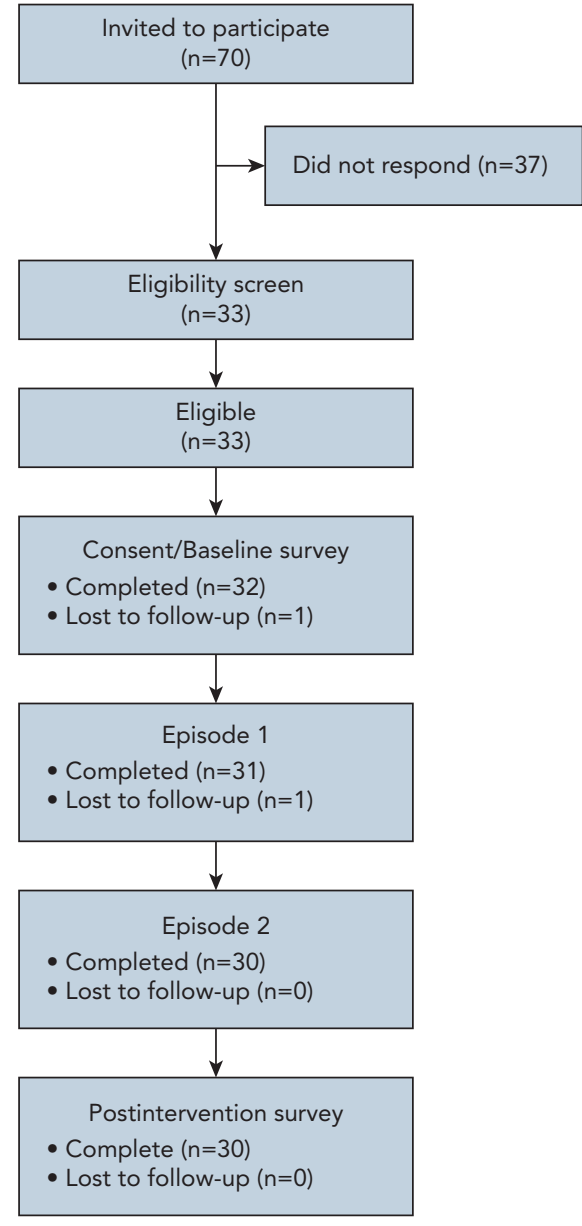

Figure 1. Study flow diagram.

study completion (94\%) passed the preset feasibility benchmarks for the study. The mean length of time taken to complete the study (ie, listen to both episodes and complete all study surveys) was nearly 2 weeks (mean [SD], 13.6 [16.8] days), with most participants (87\%) completing the study within 3 weeks and 4 completing it in 1 day.

\section{Acceptability}

Acceptability data are shown in Table 2 . Of the 30 participants who completed the study, $24(80 \%)$ endorsed the acceptability items, exceeding the preset benchmark suggestive of acceptability of the intervention. Most clinicians reported that the intervention was highly relevant, informative, and easy to use, as well as being satisfied and likely to recommend it to a colleague. Most also endorsed that the intervention would likely change their practice.

\section{Intervention Effects}

Means and confidence intervals for clinicians' baseline and postintervention scores on outcome measures are shown in Table 3. Preliminary findings show notable increases, with the means and $95 \%$ confidence intervals being uniformly in the positive range. For instance, knowledge scores increased: clinicians responded correctly to an average of 8 items postintervention compared with 6 at baseline. Scores for beliefs (self-efficacy, outcome expectancies) and comfort with discussing sexual health concerns were high at baseline, especially for outcome expectancies. However, increases were nevertheless seen for all these measures, with the highest point increase being for comfort.

\section{Summary of Lessons Learned}

Results of the qualitative analysis of the postintervention survey item assessing clinicians' lessons learned from the intervention, including relevant illustrative quotes representing each theme, are shown in Table 4 . All 30 clinicians responded to this item. Five key themes of the qualitative analysis emerged reflecting overarching lessons learned. Theme 1 (Being Proactive in Discussing Sexual Issues: It's My Responsibility) centered on the key idea that it is clinicians' responsibility to broach the subject of sexual issues with their patients and that doing so gives patients permission to discuss the issue. These quotes emphasize the importance of proactively addressing this issue, rather than waiting for the patient to raise the topic.

Theme 2 (Normalize Discussions of Sexual Health) centered on the importance of assuring patients that sexual health is a normal topic and appropriate to discuss. Clinicians discussed strategies that could assure patients of this by informing patients of the nature of sexual concerns as highly common and treating sexual issues as just another aspect of health worthy of discussion.

Theme 3 (Knowing the Patients to Target) included the main ideas that all patients should receive a discussion of sexual issues, but then certain groups of patients, including those on aromatase inhibitors and ovarian suppression, require special care due to the severity and abruptness of sexual problems they often experience.

Theme 4 (Understanding How to Raise the Topic) included 3 main clinical skills for raising the topic of sexual concerns and continuing the conversation throughout the cancer trajectory.

Finally, Theme 5 (Understanding How to Respond to Sexual Concerns) included 2 key ideas, including the importance of conveying the message that options to address women's sexual problems are available, and sharing specific information to address key common sexual concerns of vaginal dryness and discomfort during sex.

\section{Discussion}

Results of this pilot study demonstrate the acceptability and feasibility of the iSHARE mLearning intervention delivered as an informational podcast series. Rates of 
Table 2. Clinicians' Evaluations of the Intervention Acceptability $(\mathbf{N}=\mathbf{3 0})$

\begin{tabular}{|c|c|c|}
\hline Acceptability Item ${ }^{a}$ & Mean [SD] & Quite a Bit/Very ${ }^{b}$ \\
\hline Satisfaction & $4.42[0.62]$ & $93 \%$ \\
\hline Informativeness & $4.32[0.68]$ & $90 \%$ \\
\hline Relevance of information & $4.75[0.37]$ & $100 \%$ \\
\hline Ease of listening & $4.63[0.41]$ & $100 \%$ \\
\hline $\begin{array}{l}\text { Likelihood of recommending } \\
\text { to colleague }\end{array}$ & $4.27[0.67]$ & $90 \%$ \\
\hline Likelihood of changing practice & $3.83[0.77]$ & $77 \%$ \\
\hline
\end{tabular}

a $1=$ not at all; $5=$ very.

${ }^{b}$ Percentages are given for those endorsing the item for at least 1 of the 2 podcast episodes.

participation (46\% of all contacted; $97 \%$ of those responding and screening eligible) surpassed our feasibility benchmark and were comparable to other clinician communication intervention studies. ${ }^{36-38}$ These rates were noteworthy in light of the time commitment being asked of the clinicians (ie, 2 half-hour podcasts, 3 study surveys) and the fact that the bulk of enrollment occurred during the COVID-19 pandemic and the ensuing societal and professional changes experienced by many clinicians. Nevertheless, a number of clinicians contacted did not respond to our invitation to participate. Although we cannot infer reasons for nonresponse, moving forward it will be important to determine how digital interventions can best be promoted as viable alternatives to more resource-intensive in-person options. In addition, nearly all clinicians who consented completed the study, suggesting that they found the material appealing enough to finish. These findings add to a growing body of literature examining podcast interventions as having a unique role in medical education ${ }^{39,40}$ and suggest that the modality could hold appeal for clinicians even when competing demands on clinicians' time and attention are substantial. Offering Continuing Medical Education (CME) credits and limiting the surveys administered to clinicians could further improve uptake, although this will need to be tested in future research. Determining how best to optimize uptake of evidence-based interventions to enhance clinical communication about sexual health concerns will be a critical step in this area of research.

The intervention was also well-received by clinicians, with high marks given for the informative, relevant content and convenient format. Moreover, relative to the traditional in-person format used in similar studies, ${ }^{18,37,41}$ these findings suggest that the mLearning approach used here is consistent with clinicians' preferences, in that it is relatively brief, focused, and easily accessible. ${ }^{21}$ The one item of the program evaluation survey assessing acceptability that was less highly endorsed was the item assessing the likely impact on clinicians' clinical practice. In the trial of this intervention in its previous iteration, ${ }^{18}$ clinicians similarly reported feeling unconvinced that the intervention would change their practice, even though an analysis of their communication during audio recorded clinic encounters suggested otherwise. Nevertheless, the findings supporting the acceptability of the intervention content and format bode well both for the success of a larger trial and for uptake in the larger population of clinicians.

Data from this pilot study suggest that this brief intervention may improve clinicians' understanding of sexual problems among patients with breast cancer (eg, symptoms, solutions) and their comfort and confidence in clinical communication regarding sexual health concerns. Their perceived expectations for these discussions (ie, outcome expectancies) also showed a positive trend. Inadequate knowledge and skills can limit breast cancer clinicians' discussions with their patients about sexual health concerns ${ }^{21}$; addressing these barriers could help chip away at this problem. ${ }^{42}$ Moreover, the takeaway messages clinicians cited for the intervention provide context to the empirical findings and suggest that clinicians gleaned a mix of both specific information and strategies and guiding principles for navigating discussions of sexual concerns throughout the breast cancer care trajectory. For instance, clinicians commented on learning about the importance of addressing sexual

Table 3. Clinicians' Baseline and Postintervention Scores on Outcome Measures

\begin{tabular}{|c|c|c|c|}
\hline \multirow[b]{2}{*}{ Measure } & \multicolumn{3}{|c|}{ Mean $(95 \% \mathrm{Cl})$} \\
\hline & $\begin{array}{l}\text { Baseline } \\
(\mathbf{N}=\mathbf{3 0})\end{array}$ & $\begin{array}{l}\text { Postintervention } \\
\quad(\mathbf{N}=\mathbf{3 0})\end{array}$ & Difference \\
\hline Knowledge $^{a}$ & $6.5(5.9-7.2)$ & $8.1(7.7-8.5)$ & $1.60(0.82-2.3)$ \\
\hline \multicolumn{4}{|l|}{ Beliefs } \\
\hline Self-efficacy ${ }^{a}$ & $7.2(6.5-7.9)$ & $8.0(7.4-8.5)$ & $0.76(0.29-1.2)$ \\
\hline Outcome expectancies $^{a}$ & $8.4(7.9-9.0)$ & $9.0(8.6-9.4)$ & $0.52(0.17-0.86)$ \\
\hline Comfort with discussing sexual health concerns ${ }^{\mathrm{a}}$ & $7.5(6.8-8.2)$ & $8.3(7.8-8.9)$ & $0.80(0.46-1.14)$ \\
\hline
\end{tabular}

aPossible range: $0-10$. 


\section{Table 4. Qualitative Themes for Clinicians' Lessons Learned From the iSHARE Intervention}

Lesson Learned

Verbatim Illustrative Quotes

Theme 1: Being proactive in discussing sexual issues (it's my responsibility)

Initiate discussions of sexual issues

"We need to make sure to open up the conversation."

"Give patients permission to talk about sexual health and side effects."

"Providers should initiate conversations about sexual health and let patients know these issues can be further addressed at any time."

Theme 2: Normalize discussions of sexual health

Assure patients that sexual health concerns are common and appropriate for discussion

"Sexual problems are common in patients with breast cancer and should be addressed proactively."

"Providers need to normalize conversations about sexual health by including it as part of the ROS as we do nausea, fatigue, etc. This invites patients to share because they are unlikely to bring concerns up unprompted."

"Allow the discussion of possible sexual health related toxicities to be as relevant and real as any other possible treatment related toxicities...."

Broaden the accepted topic of conversation

"Be open with talking about orgasm and masturbation, and not only sex."

"I should try to ask my patients about their sexual health, desire, and vaginal dryness...."

"Raising sexual side effects such as libido during the first discussion is important."

Theme 3: Knowing the patients to target

Raise the topic with all patients

"Important to ask all patients about sexual health issues."

"Give patient permission to talk about anything that may impact QoL (sexuality, intimacy) as race, age and ethnicity is not a factor, and bring up the topic...."

"Ask all patients if they are experiencing any changes in sexual function that are distressing to them."

Give special care for certain groups

"Recommend vaginal moisturizers from the beginning of $\mathrm{Al}$ or ovarian suppression therapy (rather than wait for there to be a problem)."

"Vaginal moisturizers may be needed daily in women on Als."

"The importance of vaginal moisturizers in helping patients on aromatase inhibitors."

Theme 4: Understanding how to raise the topic

Build the relationship as the foundation

for discussing sensitive topics

Raise the topic early and often

Inquire about sexual concerns and

discuss sexual adverse effects

"That is important to know about a patient's relationships (partners, etc.) to develop trust and comfort to best address sexual concerns."

"Loved the tip on using the social history as a way to get to know patients and their partners and that this is an excellent segue to discussing sexual side effects of treatment."

"Sexual issues in women with breast cancer need to be discussed openly, and there are resources out there for women and physicians."

"It is important to introduce the topic early and revisit as the patient seems receptive...."

"That I need to include more information regarding sexual health at the outset of a woman's treatment plan to lay the foundation for an open discussion regarding her sexual concerns throughout treatment and continuing through survivorship."

"Talk about sexual health often and up front in the cancer journey."

"Simply asking a patient if she has any sexual concerns is an easy way to open up the conversation."

"Even acknowledging sexual side effects goes a long way."

"Discuss sexual side effects as part of routine tox evaluation and become more

knowledgeable about management of sexual side effects to advise patient."

Theme 5: Understanding how to respond to sexual concerns

Convey the message that help is available

Address key concerns: vaginal health issues

"Important to discuss sexual issues up front and assure patients that help is available."

"... Options to help exist."

"... There are resources out there for women and physicians."

"The importance of vaginal moisturizers in helping patients on Als."

"Vaginal moisturizers should be used 3 to 5 times a week"

"Vaginal moisturizers may be needed daily in women on Als."

Abbreviations: Al, aromatase inhibitor; iSHARE, improving Sexual Health and Augmenting Relationships through Education; QoL, quality of life; ROS review of systems.

health concerns in all women with a breast cancer diagnosis and that they can build on the patient-provider relationship as a foundation for discussing these issues early and often. These points are consistent with recommendations from the NCCN Clinical Practice Guidelines in Oncology (NCCN
Guidelines) for Survivorship: Sexual Function ${ }^{11}$ and with patients' wishes for such discussions. ${ }^{13,21}$

This study had several strengths. First, the mLearning intervention we tested is an innovative approach to addressing the lack of discussion regarding sexual 
concerns among women with breast cancer, which could potentially be disseminated more easily and with fewer resources compared with interventions using traditional trainer-based methods. Second, we used both quantitative and qualitative assessments to understand the impact of the intervention for clinicians and collected detailed information to understand feasibility and acceptability. Finally, the sample included both physicians and advanced practice clinicians, which could facilitate larger-scale testing in this population and bolster the generalizability of the intervention to a wide range of breast cancer clinicians.

To maintain consistency with the initial iSHARE intervention, the mLearning adaptation described here was tested in breast cancer clinicians in a medical oncology setting; however, surgeons and radiation oncologists could also benefit from evidence-based information on discussing sexual health concerns, and efforts should expand to include these groups. Future studies should also aim to include a more diverse sample of clinicians with respect to racial and ethnic background and consider how best to address differences in patient health literacy when discussing sexual health concerns. The study also had other limitations, including no assessment of clinicians' communication about sexual health concerns, longterm follow-up, or control group. We are planning a larger trial that will address these concerns and evaluate intervention effects on clinicians' communication behaviors through coding dialogue. Given that trials of online interventions in medical education have not always yielded consistent results, ${ }^{43}$ a well-designed trial will be critical. We also left the time frame for completing the study open so that clinicians could choose when to listen to the podcasts according to their availability and preferences. This may have helped bolster completion rates, but also created a range of time frames in which clinicians finished the study. In an efficacy trial, it will be important to set time limits on intervention use so that intervention effects can reliably be determined.

\section{Conclusions}

This mLearning (iSHARE) intervention holds promise as a tool to hasten patient-clinician communication about sexual health concerns among patients with breast cancer. Offering CMEs and using organizations such as the NCCN or ASCO could potentially enhance reach for this intervention. ${ }^{44}$ We hope that this study will help close the gap on patient-provider communication about sexual health concerns.

\section{Acknowledgments}

We wish to thank the breast cancer clinicians who participated in our research.

Submitted January 21, 2021; final revision received February 26, 2021; accepted for publication March 2, 2021.

Published online August 13, 2021.

Author contributions: Study concept and design: Reese, Zimmaro, Bober, Sorice, Handorf, Wittenberg, Beach, Wolff, Daly, Lepore. Data acquisition: Reese, Zimmaro, Bober, Sorice, El-Jawahri. Data analysis and interpretation: Reese, Zimmaro, Bober, Sorice, Handorf, Izquierdo, Lepore. Manuscript preparation: Reese, Zimmaro, Handorf, Lepore. Manuscript review and editing: All authors

Disclosures: The authors have disclosed that they have not received any financial consideration from any person or organization to support the preparation, analysis, results, or discussion of this article.

Funding: Research reported in this publication was supported by the $\mathrm{NCl}$ of the $\mathrm{NIH}$ under award numbers R03CA235238, P30CA006927, and T32-CA-0090354

Disclaimer: The content is solely the responsibility of the authors and does not necessarily represent the official views of the $\mathrm{NIH}$.

Correspondence: Jennifer Barsky Reese, PhD, Fox Chase Cancer Center, 333 Cottman Avenue, 4th Floor, Young Pavilion, Philadelphia, PA 19111. Email: Jennifer.Reese@fccc.edu

\section{References}

1. Fobair P, Spiegel D. Concerns about sexuality after breast cancer. Cancer J 2009;15:19-26.

2. Fobair $P$, Stewart $S L$, Chang $S$, et al. Body image and sexual problems in young women with breast cancer. Psychooncology 2006;15:579-594.

3. Avis NE, Crawford S, Manuel J. Quality of life among younger women with breast cancer. J Clin Oncol 2005;23:3322-3330.

4. Baucom DH, Porter LS, Kirby JS, et al. Psychosocial issues confronting young women with breast cancer. Breast Dis 2005-2006;23:103-113.

5. Ganz PA, Coscarelli A, Fred C, et al. Breast cancer survivors: psychosocial concerns and quality of life. Breast Cancer Res Treat 1996;38:183-199.

6. Melisko ME, Goldman M, Rugo HS. Amelioration of sexual adverse effects in the early breast cancer patient. J Cancer Surviv 2010;4:247-255.

7. Bober SL, Varela VS. Sexuality in adult cancer survivors: challenges and intervention. J Clin Oncol 2012;30:3712-3719.

8. Ganz PA, Desmond KA, Leedham B, et al. Quality of life in long-term, disease-free survivors of breast cancer: a follow-up study. J Natl Cancer Inst 2002;94:39-49.

9. Runowicz CD, Leach CR, Henry NL, et al. American Cancer Society/American Society of Clinical Oncology breast cancer survivorship care guideline. CA Cancer J Clin 2016;66:43-73.

10. Carter J, Lacchetti C, Andersen BL, et al. Interventions to address sexual problems in people with cancer: American Society of Clinical Oncology clinical practice guideline adaptation of Cancer Care Ontario guideline. J Clin Oncol 2018;36:492-511.

11. Denlinger CS, Sanft T, Baker KS, et al. Survivorship, Version 2.2018, NCCN Clinical Practice Guidelines in Oncology. J Natl Compr Canc Netw 2018; 16:1216-1247. To view the most recent version, visit NCCN.org

12. Hill EK, Sandbo S, Abramsohn E, et al. Assessing gynecologic and breast cancer survivors' sexual health care needs. Cancer 2011;117:2643-2651.

13. Flynn KE, Reese JB, Jeffery DD, et al. Patient experiences with communication about sex during and after treatment for cancer. Psychooncology 2012;21:594-601.

14. Reese JB, Sorice K, Beach MC, et al. Patient-provider communication about sexual concerns in cancer: a systematic review. J Cancer Surviv 2017; 11:175-188.

15. Dyer K, das Nair R. Why don't healthcare professionals talk about sex? A systematic review of recent qualitative studies conducted in the United kingdom. J Sex Med 2013;10:2658-2670.

16. Hautamäki K, Miettinen M, Kellokumpu-Lehtinen PL, et al. Opening communication with cancer patients about sexuality-related issues. Cancer Nurs 2007;30:399-404. 
17. Albers LF, Palacios LAG, Pelger RCM, et al. Can the provision of sexua healthcare for oncology patients be improved? A literature review of educational interventions for healthcare professionals. J Cancer Surviv 2020;14:858-866.

18. Reese JB, Lepore SJ, Daly MB, et al. A brief intervention to enhance breast cancer clinicians' communication about sexual health: feasibility, acceptability, and preliminary outcomes. Psychooncology 2019;28:872-879.

19. Harris PA, Taylor R, Thielke R, et al. Research electronic data capture (REDCap)—a metadata-driven methodology and workflow process for providing translational research informatics support. J Biomed Inform 2009;42:377-381.

20. Harris PA, Taylor R, Minor BL, et al. The REDCap consortium: building an international community of software platform partners. J Biomed Inform 2019;95:103208.

21. Reese JB, Beach MC, Smith KC, et al. Effective patient-provider communication about sexual concerns in breast cancer: a qualitative study. Support Care Cancer 2017;25:3199-3207.

22. Skinner CS, Pollak KI, Farrell D, et al. Use of and reactions to a tailored CD-ROM designed to enhance oncologist-patient communication: the SCOPE trial intervention. Patient Educ Couns 2009;77:90-96.

23. Cabana MD, Rand CS, Powe NR, et al. Why don't physicians follow clinical practice guidelines? A framework for improvement. JAMA 1999;282:14581465.

24. Merckaert I, Libert Y, Razavi D. Communication skills training in cancer care: where are we and where are we going? Curr Opin Oncol 2005;17:319-330.

25. Bandura A. Social foundations of thought and action: a social cognitive theory. Englewood Cliffs, NJ: Prentice Hall, Inc; 1986.

26. Graves KD. Social cognitive theory and cancer patients' quality of life: a meta-analysis of psychosocial intervention components. Health Psychol 2003;22:210-219.

27. Graves KD, Carter CL, Anderson ES, et al. Quality of life pilot intervention for breast cancer patients: use of social cognitive theory. Palliat Support Care 2003;1:121-134

28. Kravitz RL, Tancredi DJ, Street RL, Jr., et al. Cancer Health Empowerment for Living without Pain (Ca-HELP): study design and rationale for a tailored education and coaching intervention to enhance care of cancer-related pain. BMC Cancer 2009;9:319

29. Zimmaro LA, Izquierdo B, Beach MC, et al. Developing a mobile technology-based (mLearning) intervention to enhance breast cancer clinicians' communication about sexual health: results from formative interviews. Presented at the 7th Conference of the Scientific Network on Female Sexua Health and Cancer; November 13-15, 2019; Columbus, Ohio.
30. Wittenberg E, Ferrell B, Goldsmith J, et al. COMFORT ${ }^{\mathrm{TMSM}}$ communication for oncology nurses: program overview and preliminary evaluation of a nationwide train-the-trainer course. Patient Educ Couns 2018;101:467-474.

31. Goldsmith JV, Wittenberg E, Ferrell B. An app to support difficult interactions among providers, patients, and families. J Adv Pract Oncol 2015;6: 481-485.

32. Wittenberg-Lyles E, Goldsmith J, Ferrell B, et al. Assessment of an interprofessional online curriculum for palliative care communication training. J Palliat Med 2014;17:400-406.

33. Bandura A. Guide for constructing self-efficacy scales. In: Pajares F, Urdan T, eds. Self-Efficacy Beliefs of Adolescents. Greenwich, CT: IAP-Information Age Publishing Inc; 2006:307.

34. Wittenberg E, Ferrell B, Kanter E, et al. Health literacy: exploring nursing challenges to providing support and understanding. Clin J Oncol Nurs 2018;22:53-61.

35. Freedland KE. Pilot trials in health-related behavioral intervention research problems, solutions, and recommendations. Health Psychol 2020:39:851-862.

36. Fujimori $M$, Shirai $Y$, Asai $M$, et al. Effect of communication skills training program for oncologists based on patient preferences for communication when receiving bad news: a randomized controlled trial. J Clin Oncol 2014 32:2166-2172.

37. Wang LY, Pierdomenico A, Lefkowitz A, et al. Female sexual health training for oncology providers: new applications. Sex Med 2015;3:189197.

38. Tulsky JA, Arnold RM, Alexander SC, et al. Enhancing communication between oncologists and patients with a computer-based training program: a randomized trial. Ann Intern Med 2011;155:593-601.

39. Berk J, Trivedi SP, Watto M, et al. Medical education podcasts: where we are and questions unanswered. J Gen Intern Med 2020;35: 2176-2178.

40. Little A, Hampton Z, Gronowski T, et al. Podcasting in medicine: a review of the current content by specialty. Cureus 2020;12:e6726.

41. Winterling J, Lampic C, Wettergren L. Fex-Talk: a short educational intervention intended to enhance nurses' readiness to discuss fertility and sexuality with cancer patients. J Cancer Educ 2020;35:538-544.

42. Reese JB, Bober SL, Daly MB. Talking about women's sexual health after cancer: why is it so hard to move the needle? Cancer 2017;123:4757-4763.

43. Vaona A, Banzi R, Kwag KH, et al. E-learning for health professionals. Cochrane Database Syst Rev 2018;1:CD011736.

44. Pender LK, Caracio R. Assessing community physician ability to identify and manage immune-mediated adverse events (imAEs) through online continuing medical education (CME) [abstract]. J Clin Oncol 2019;37(Suppl):Abstract 78. 